\title{
Short-Course Versus Long-Course Systemic Antibiotic Treatment for Uncomplicated Intravascular Catheter- Related Bloodstream Infections due to Gram-Negative Bacteria, Enterococci or Coagulase-Negative Staphylococci: A Systematic Review
}

\author{
Severin Muff · Alexis Tabah · Yok-Ai Que · Jean-François Timsit • \\ Leonard Mermel · Stephan Harbarth · Niccolò Buetti (D)
}

Received: March 29, 2021 / Accepted: May 22, 2021 / Published online: June 25, 2021

(c) The Author(s) 2021

\section{ABSTRACT}

Introduction: The optimal duration of systemic antimicrobial treatment for catheter-related bloodstream infections (CRBSI) is unknown. In

Supplementary Information The online version contains supplementary material available at https:// doi.org/10.1007/s40121-021-00464-0.

S. Muff · S. Harbarth · N. Buetti $(\bowtie)$ Infection Control Program and WHO Collaborating Centre on Patient Safety, University of Geneva Hospitals and Faculty of Medicine, Rue GabriellePerret-Gentil 4, 1205 Geneva, Switzerland e-mail: niccolo.buetti@gmail.com

A. Tabah

ICU, Faculty of Medicine, Redcliffe Hospital, University of Queensland, Brisbane, QLD, Australia

Y.-A. Que

Inselpital, Bern University Hospital, University of Bern, Bern, Switzerland

\section{J.-F. Timsit}

Team DeSCID, University of Paris, INSERM IAME, U1137, Paris, France

\section{J.-F. Timsit}

Medical and Infectious Diseases Intensive Care Unit, AP-HP, Bichat-Claude Bernard University Hospital, 46 rue Henri Huchard, 75877 Paris Cedex, France

\section{Mermel}

Division of Infectious Diseases, Department of Medicine, Rhode Island Hospital, Alpert Medical School of Brown University, 593 Eddy St., Providence, RI, USA this systematic review, we aimed to assess the efficacy of short-course treatment for CRBSI due to Gram-negative bacteria, coagulase-negative staphylococci and enterococci.

Methods: We systematically searched the electronic bibliographic databases MEDLINE, EMBASE and Cochrane Library for studies published before February 2021. All studies that investigated the duration of adequate systemic antibiotic treatment in adult patients with uncomplicated intravascular catheter infections due to Gram-negative bacteria, coagulase-negative staphylococci or enterococci were eligible for inclusion. Studies including concomitant treatment with antibiotic lock therapy were excluded. The primary outcomes were clinical failure/cure, mortality and microbiologic-confirmed relapse.

Results: Seven retrospective cohort studies and one case-cohort study met the inclusion criteria. No randomized controlled studies met inclusion criteria. The quality of the included studies was low $(n=7)$ to moderate $(n=1)$. No significant differences were observed regarding mortality and microbiological relapse between short-course and long-course systemic antibiotic treatment in patients with CRBSI due to coagulase-negative staphylococci or Gram-negative bacteria. No association was found between mortality and treatment duration in the two studies assessing enterococcal CRBSI.

Conclusion: The limited data available suggests that shorter systemic antibiotic treatment 
duration may be sufficient for uncomplicated CRBSI. Further well-designed prospective studies are needed to confirm these findings.

Trial Registration Number: CRD42021224946 (PROSPERO)

Keywords: Catheter-related bloodstream infection; Coagulase-negative staphylococci; CRBSI; Enterococcal; Enterococcus; Gramnegative; Intravascular catheter infection

\section{Key Summary Points}

The optimal duration of antibiotic therapy for intravascular catheter-related bloodstream infections is unknown and current recommendations are mainly based on expert opinions.

The aim of this systematic review was to assess short-course versus long-course treatment for catheter-related bloodstream infections due to Gramnegative bacteria, enterococci or coagulase-negative staphylococci.

In the studies assessed, patients with short-course treatment had similar mortality rates, clinical cure rates and microbiological relapse rates as patients with long-course treatment.

Prospective studies are needed to determine the optimal antimicrobial treatment duration for catheter-related bloodstream infections.

\section{DIGITAL FEATURES}

This article is published with digital features, including a summary slide, to facilitate understanding of the article. To view digital features for this article go to https://doi.org/10.6084/ m9.figshare.14627559.

\section{INTRODUCTION}

Intravascular catheters are used for the administration of fluids, blood products and medication, to monitor haemodynamic parameters and for haemodialysis. They are essential for the care of hospitalized patients, particularly in the case of critical illness. Unfortunately, intravascular catheters are also possible ports of entry for pathogens into the bloodstream. In 2019, over 18,000 cases of central line associated bloodstream infections (CLABSI) were reported in acute care hospitals in the USA [1]. In Europe, $36.5 \%$ of intensive care unit (ICU)-acquired bloodstream infections (BSIs) were linked to intravascular catheters and rates of CLABSI ranged from 1.7 to 4.8 episodes per 1000 catheter days [2]. Catheter-related bloodstream infections (CRBSI) were associated with substantial morbidity, increased hospital length of stay [3, 4], mortality $[4,5]$ and an estimated attributable cost of US $\$ 45,814$ per event [6]. According to recently published Centers for Disease Control and Prevention (CDC) data, Gram-negative bacteria (GNB), enterococci and coagulase-negative staphylococci (CoNS) were among the most frequently detected bacterial pathogens associated with such infections [7].

Research to determine the appropriate antibiotic treatment duration is part of the United States National Action Plan for Combating Antibiotic-Resistant Bacteria 2020-2025 [8]. Recent literature derived from randomized controlled trials (RCTs) suggested that shortcourse treatment for hospital and communityacquired Gram-negative bloodstream infections is non-inferior to longer courses of treatment $[9,10]$. Similar results were confirmed by two recent systematic reviews $[11,12]$. Nevertheless, little is known about the optimal duration of antimicrobial treatment for CRBSI. The Infectious Disease Society of America (IDSA) and other current guidelines recommended treatment durations of 5-14 days for uncomplicated enterococcal CRBSI, 7-14 days for Gram-negative CRBSI and 5-7 days for CoNS-CRBSI [13-16]. However, these recommendations were mainly based on expert opinions and, to our knowledge, no recent literature review has been 
performed. Therefore, we conducted a systematic review with the objective to evaluate if the duration of systemic antibiotic treatment in patients with uncomplicated CRBSI due to GNB, enterococci, or CoNS influenced the rates of mortality, clinical cure/failure or microbiological relapse.

\section{METHODS}

This study was performed according to the Preferred Reporting Items for Systematic reviews and Meta-Analyses (PRISMA) statements and was prospectively registered within the international prospective register of systematic reviews (PROSPERO, ID CRD42021224946) on 15 December 2020 [17]. This article is based on previously conducted studies and does not contain any new studies with human participants or animals performed by any of the authors.

\section{Search Strategy, Study Screening and Selection}

We conducted a systematic search of the electronic bibliographic databases MEDLINE (Pubmed, 1949 to date), EMBASE (Elsevier, 1974 to date) and the Cochrane Library (including the Cochrane Central Register of Controlled Trials). Additionally, we manually searched throughout the European Congress of Clinical Microbiology and Infectious Diseases (ECCMID 2008-2019) and ID-WEEK (2014-2019) conference websites and clinicaltrials.gov to detect grey literature and unpublished studies. The full search strategies are available in the supplementary material. Briefly, the searches were constructed with controlled vocabulary terms and keywords describing "intravascular catheter infection", "antibiotic therapy", "duration of therapy", "Gram-negative bacilli", "enterococcus" and "coagulase-negative staphylococci". The controlled vocabulary terms were adapted for each database. We placed no restrictions on language, publication status or publication period. The final search update was performed on 15 February 2021. The results were imported into a literature management software to exclude duplicate publications. Then, title and abstract screening and subsequently full-text screening were performed independently by two authors (Severin Muff and Niccolò Buetti), with any disagreement resolved through discussion between the authors. To manage the selection process, we used a web-based screening and data extraction tool [18]. All study types with the exception of case reports, letters and comments were eligible for inclusion.

We included studies that investigated the duration of adequate antibiotic treatment in adult patients with uncomplicated intravascular catheter infections due to GNB, enterococci or CoNS. Studies assessing treatment duration for other microorganisms (e.g. Staphylococcus aureus or Candida species) were excluded because they necessitate longer antibiotic treatment durations (i.e. at least 14 days). Of note, Staphylococcus lugdunensis was not included among CoNS since it can cause an aggressive clinical course, and as a consequence, longer treatment durations are recommended $[15,16,19,20]$. We excluded studies investigating lock therapies with systemic antimicrobials because lock therapy may require more prolonged systemic antibiotic courses, and our target population was uncomplicated intravascular catheter infection. Intravascular catheter infections were defined as uncomplicated if clinical and microbiological signs of infections resolved within $72 \mathrm{~h}$ after initiation of antibiotic treatment and if complications like metastatic infection, endocarditis or suppurative thrombophlebitis were absent. Studies including antimicrobial-coated catheters were excluded. Studies exclusively including paediatric patients were excluded. Studies with no age restrictions were included if the majority of the study population was adult.

\section{Data Extraction}

For evidence synthesis we used a standardized form to extract data from the included studies. We extracted data on study design, setting, participants, intervention groups (i.e. treatment duration), catheter removal and outcome 
measurements. Information about risk of bias assessment was extracted with a separate form.

\section{Intervention and Outcomes}

The interventions assessed by this review were short-course versus long-course adequate antimicrobial treatment for uncomplicated intravascular catheter-related infections due to GNB, CoNS or enterococci. Duration of shortand long course treatment was predefined by the authors of the selected studies. Primary outcomes were mortality (in-hospital or day-30 mortality), clinical cure/clinical failure and microbiologically confirmed relapse. Clinical cure was defined as resolution of symptoms and/or the significant reduction in inflammatory parameters. Clinical failure was defined as persistence of sign and symptoms of infections and/or lack of significant reduction in inflammatory parameters. We assumed that patients not fulfilling the criteria for clinical failure were 'clinically cured'. Microbiological relapse was defined as reoccurrence of the same pathogen in at least one blood culture after an initial clinical response to treatment within the follow-up period. Secondary outcomes were antibiotic-associated adverse events.

\section{Risk of Bias}

To assess the quality (including the risk of bias) of cohort and case-control studies we used the Newcastle-Ottawa scale [21]. The studies were evaluated by two authors (Severin Muff and Niccolò Buetti) independently; any disagreement was resolved through discussion. Scores of 0-3, 4-6 and 7-9 were considered as low, moderate and high quality, respectively.

\section{Study Synthesis}

Since only retrospective observational studies with high levels of clinical and methodological heterogeneity were retrieved, we did not perform a quantitative synthesis of data and we summarized our findings in the form of a narrative synthesis.

\section{RESULTS}

\section{Selection}

The review process is summarized in Fig. 1. Our search strategy identified 1491 studies, of which 236 duplicates were removed. Of the remaining 1255 studies, 11 were potentially eligible. After full-text screening, three of 11 studies were excluded because of insufficient assessment of treatment duration $(n=2)$ and missing assessment of intravascular catheter infections $(n=1)$. Finally, eight studies were included in the review.

\section{Study Characteristics}

The main characteristics of the included studies are presented in Table 1. Among eight included studies, seven were cohort studies [22-28]. One study described a retrospective cohort (i.e. "phase 1") and a prospective case-cohort (i.e. "phase 2 "). The cases in the phase 2 were matched with controls of phase 1 and we only included the results of the case-cohort phase [29]. The total number of included patients was 780 with numbers ranging from 44 to 188 patients per study. All studies were conducted in tertiary-care hospitals and only hospitalized patients were included. All cohort studies assessed the whole database of their institutions to identify patients fulfilling the inclusion criteria. Adult patients were included in four studies [24, 26, 27, 29], whereas four studies included patients of all ages [22, 23, 25, 28]. Catheter removal was mandatory for inclusion in five studies [22-24, 27, 29]. No study assessed antibiotic-associated adverse events.

The risk of bias was assessed by the Newcastle-Ottawa scale [21] (Table 2). According to our study assessment seven studies [23-29] were of low methodological quality while one study [22] was of moderate quality.

\section{Gram-Negative Intravascular Catheter Infections}

Three studies, including a total of 157 patients, assessed treatment duration for uncomplicated 

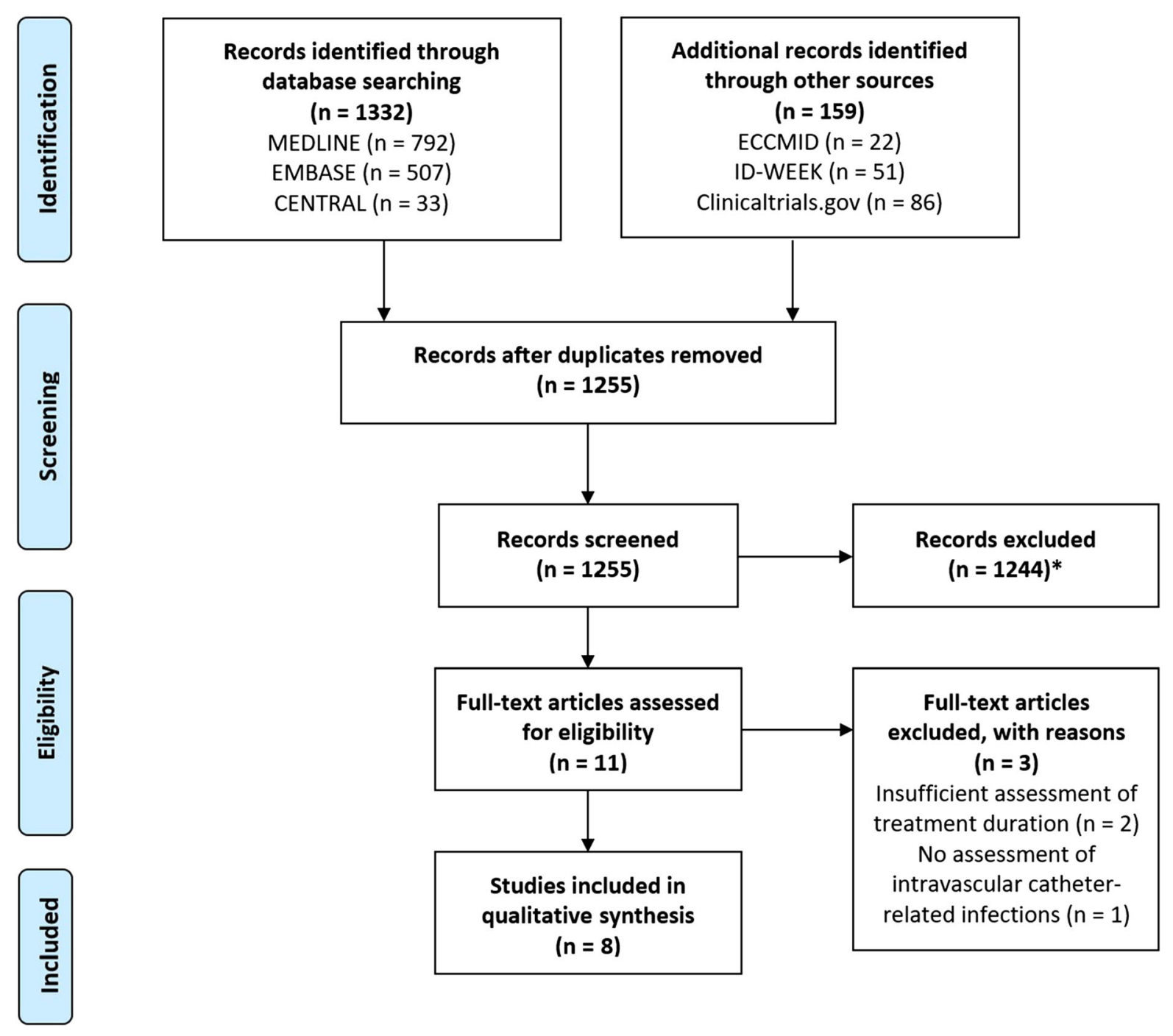

Fig. 1 Study flow diagram. ${ }^{*}$ Studies did not meet inclusion criteria

intravascular catheter-related infections due to Gram-negative bacteria [22, 24, 29]. In all of these studies, catheter removal was mandatory for inclusion. Two cohort studies compared up to 7 days to more than 7 days of treatment $[22,24]$. The differences between the short- and long-course group for clinical cure, relapse and 30-day mortality were not significantly different in both studies. The case-cohort study compared patients receiving at least 14 days with patients receiving 7 days of antibiotic treatment [29]. No significant differences between the treatment groups regarding death and relapse were observed. Clinical cure was not investigated.

\section{CoNS Intravascular Catheter Infections}

Three cohort studies compared short- versus long-course treatment for CRBSI due to coagulase-negative staphylococci $[23,27,28]$. Treatment durations varied from no antibiotic treatment to less than 10 days of treatment in the short-course groups and from more than 3 days to more than 10 days in the long-course groups.

The two more recent studies were relatively similar: Hebeisen et al. compared no antibiotic treatment with treatment for at least 5 days [27] and San-Juan et al. compared at most 3 days 


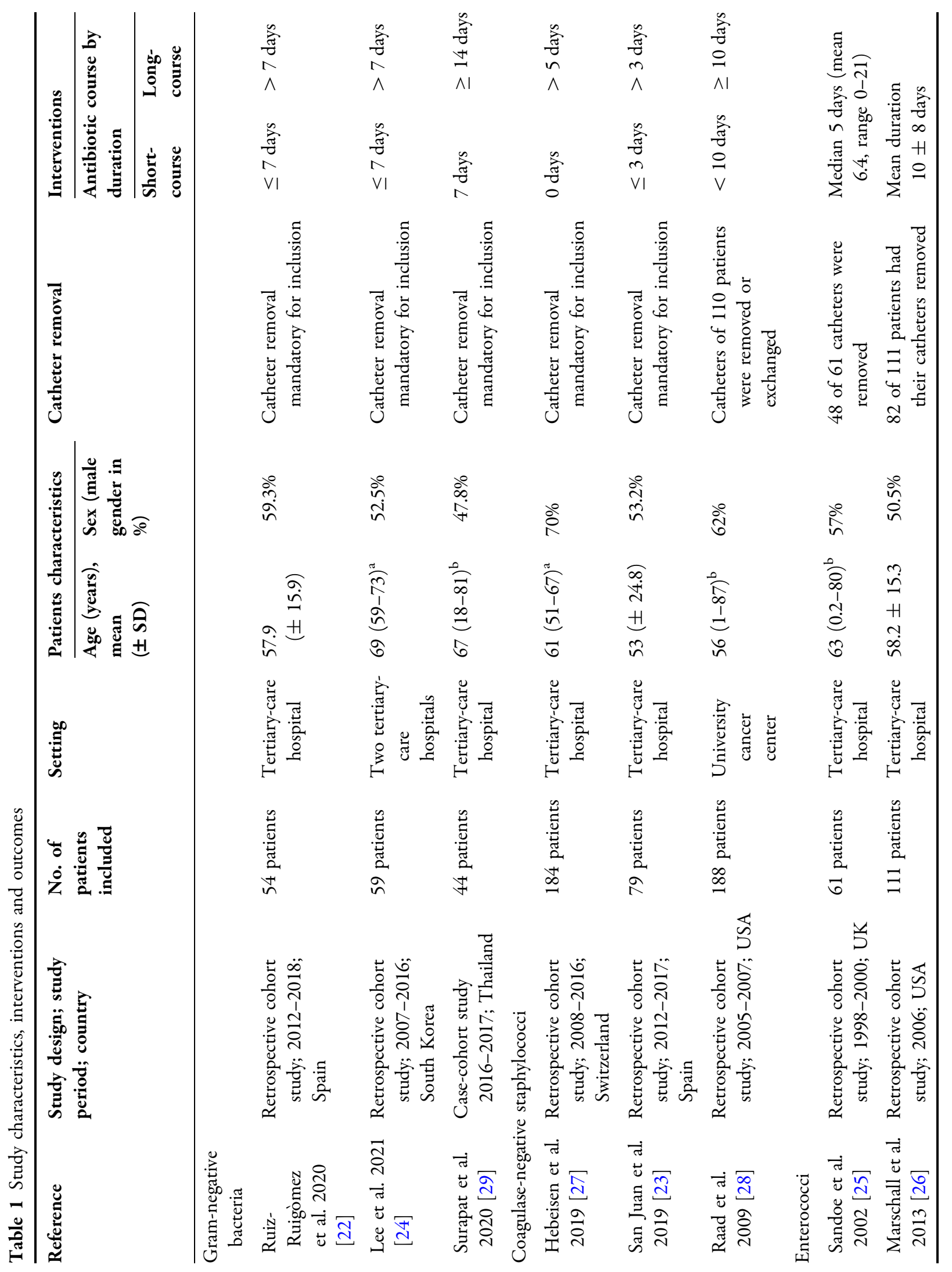




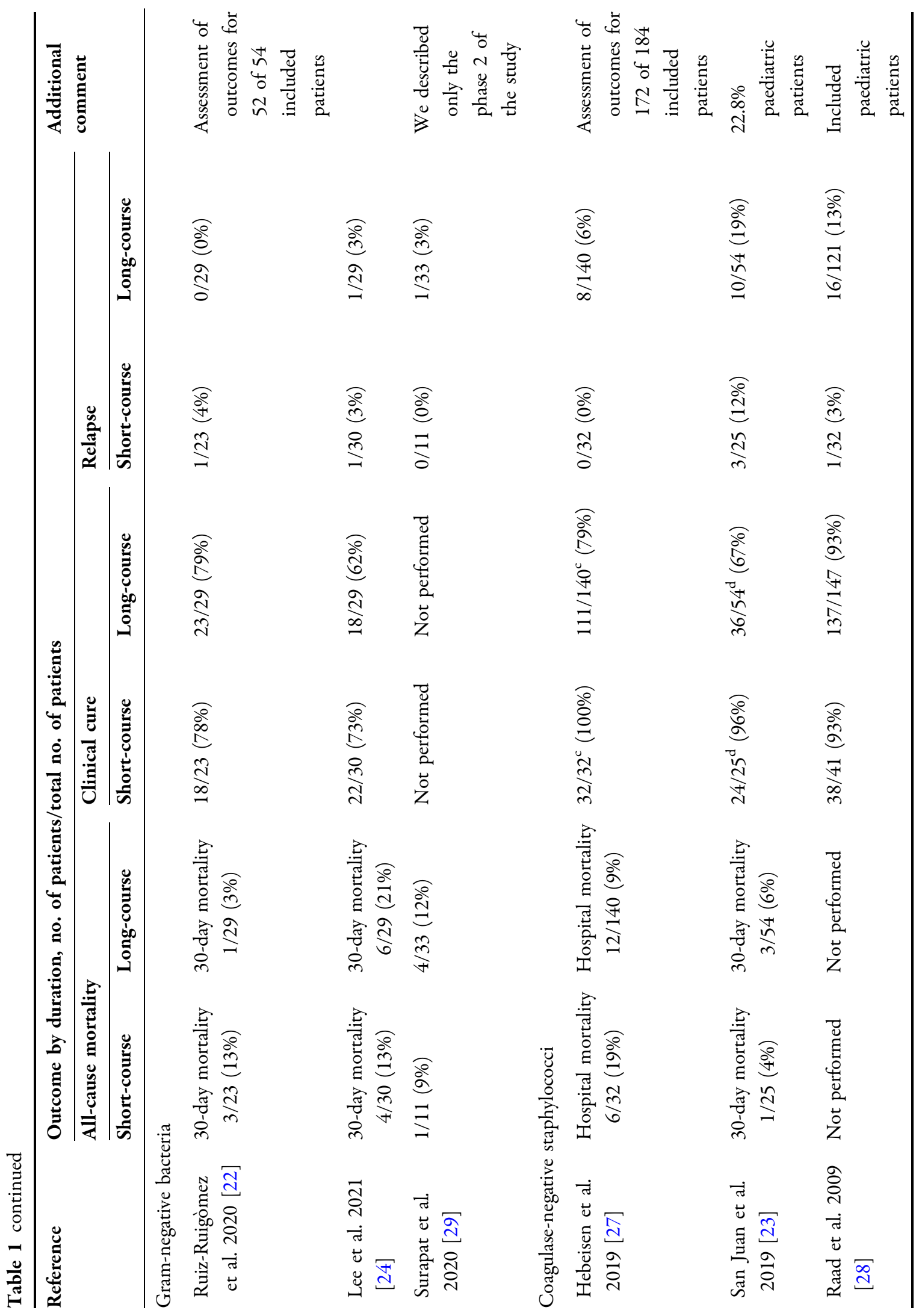




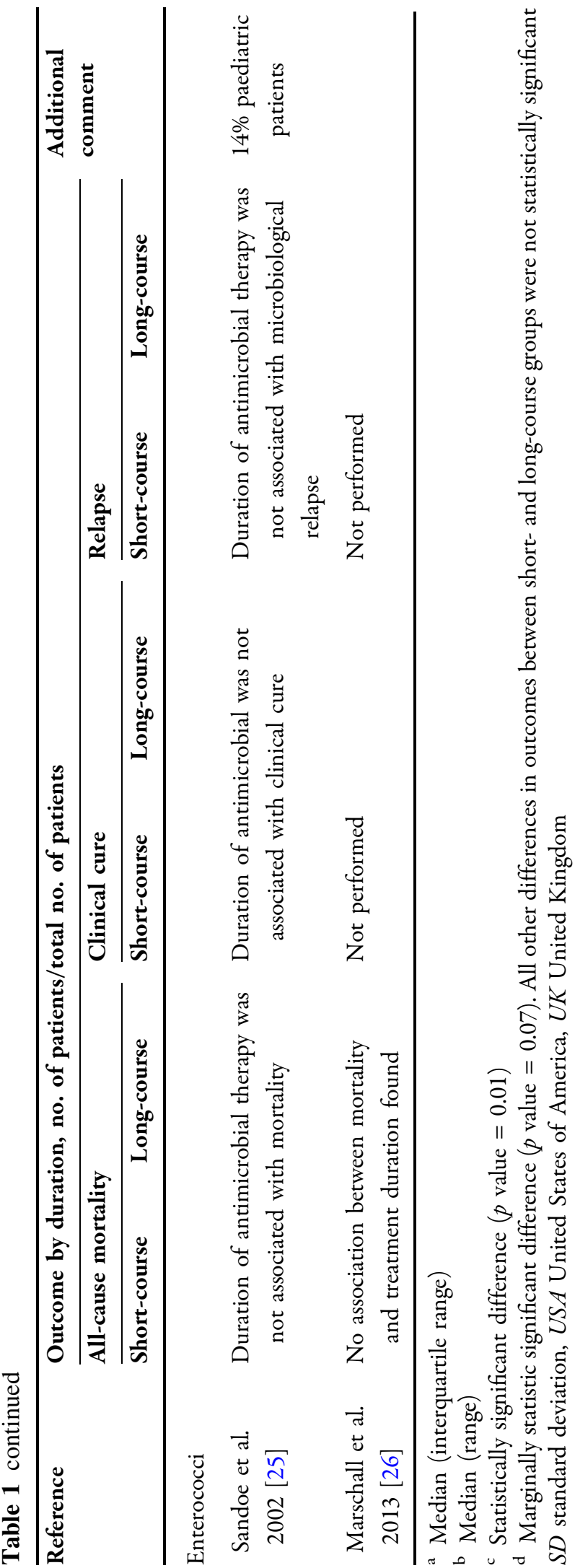

(which included patients with no active antibiotic treatment) with more than 3 days of treatment [23]. Catheter removal was mandatory in these two studies. The number of patients with clinical cure was significantly lower in the long-course treatment groups in both studies. Hebeisen et al. reported that all patients with short-course treatment (32/32) were clinically cured in contrast to only $79 \%$ $(111 / 140)$ in the long-course group $(P=0.01)$. San-Juan et al. reported similar results with $96 \%$ $(24 / 25)$ clinically cured patients in the shortcourse treatment group and only 67\% (36/64) clinically cured patients in the long-course treatment group $(P=0.07)$. However, patients in the long-course groups were significantly more immunocompromised [27] or more severely ill [23]. No significant differences in relapse or mortality were found. The authors concluded that catheter removal may be sufficient to treat CoNS-CRBSI in selected patients.

Raad et al. primarily assessed catheter retention versus removal and included only patients with cancer [28]. The study revealed similar clinical cure and relapse rates for prolonged therapy (i.e. at least 10 days) and shorter therapy durations (i.e. less than 10 days).

\section{Enterococcal Intravascular Catheter Infections}

Only two cohort studies investigated enterococcal CRBSI $[25,26]$. Both primarily investigated catheter retention versus catheter removal and/or catheter management. Therefore, patients with catheter retainment were included in both studies. No studies performed an exhaustive comparison between short versus and longer antimicrobial treatment duration. Treatment duration was evaluated using univariate analyses. The first study assessed 61 enterococcal CRBSIs. Antimicrobial treatment duration did not impact cure, treatment failure or relapse [25]. Moreover, an analysis of patients treated by catheter removal plus adequate antibiotic therapy and patients treated by catheter removal without (adequate) antibiotic therapy did not show statistically significant differences for these outcomes. The second 


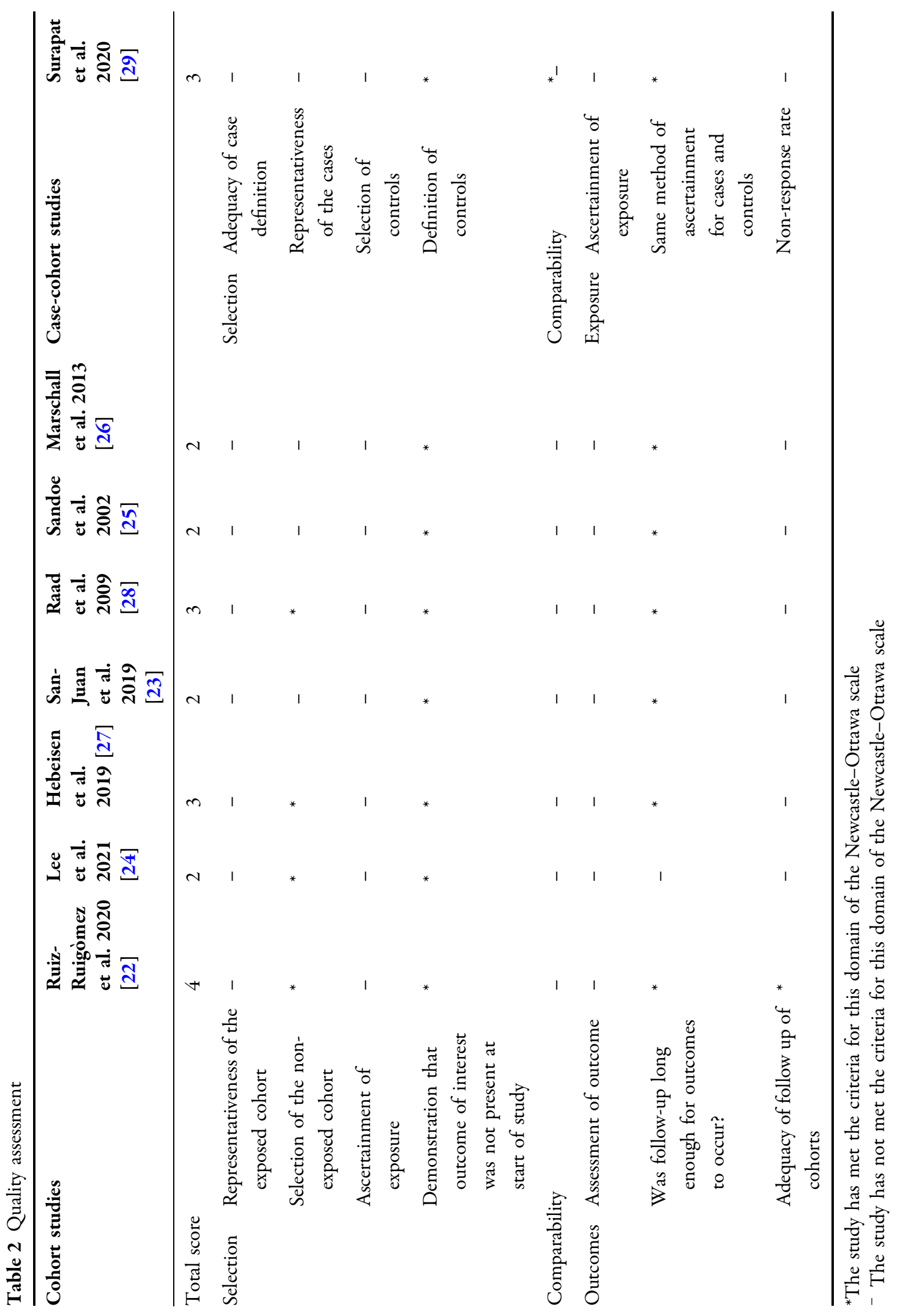


study included 111 enterococcal CRBSIs and did not find an association between the treatment duration and mortality [26].

\section{DISCUSSION}

Over the last 2 decades, a substantial reduction in CLABSI rates has been achieved owing to the development and implementation of effective prevention measures [30]. Our systematic review identified only eight observational studies which assessed treatment duration for Gram-negative, CoNS and enterococcal CRBSI. Interestingly, no RCT that compared shortcourse versus long-course antibiotic treatment durations for intravascular catheter infections was retrieved. High-quality research on the management of intravascular catheter infections is scarce and guidelines rely on expert opinion. The few studies addressing the topic included a very limited number of patients and, probably because of the retrospective nature of these studies, short- and long-course groups were not well balanced. Notwithstanding, they did not identify significant differences in mortality or relapse rates between short- and longcourse treatment groups.

Optimizing use of antibiotics is an essential step in fighting against antibiotic resistance [8]. Our results revealed that unadjusted outcomes for short-course treatment (i.e. 7 days) and longer courses of treatment were similar among uncomplicated Gram-negative CRBSI $[22,24,29]$. Our findings were supported by two recent RCTs on treatment of uncomplicated Gram-negative bacteraemia including catheterrelated urinary tract infection, which demonstrated similar outcomes for short- and longcourse antibiotic therapy $[9,10]$. In light of these considerations, a treatment duration of 7 days after source control (i.e. catheter removal) is a reasonable recommendation for Gram-negative CRBSI.

CoNS, traditionally considered typical skin commensals, have emerged as common causes of nosocomial infections [31]. International guidelines recommended a treatment duration of 5-7 days for CoNS-CRBSI [14-16]. The treatment durations were shorter than those recommended (i.e. 0 days and less than 3 days) in two of the studies we included in this review [14-16, 23, 27]. Relapse and mortality rates in the study groups without treatment or with short treatment duration were low. Curiously, the clinical cure rate was significantly lower in the long-course groups [23, 27]; however, patients in the long-course groups were more predisposed to an unfavourable course (i.e. immunosuppressed, haematological cancer, higher Pitt bacteraemia score). Overall, a very short-course (i.e. at most 3 days) of antibiotic treatment should be therefore considered for CoNS-CRBSI. The risk of unfavourable clinical courses in patients with severe underlining conditions is most likely considerably higher and they therefore may need longer antibiotic therapy durations.

Little is known about the optimal duration of treatment for enterococcal intravascular catheter infections. The lack of association between treatment duration and mortality, as well as clinical cure and microbiological relapse in one study [25], implied that longer treatment did not improve outcomes and short treatment duration may be considered for some patients with this infection. However, RCTs assessing the optimal treatment duration are needed to develop well-founded recommendations.

Current guidelines and expert statements recommended treatment durations of 7-14 days for Gram-negative CRBSI, 5-7 days for CoNS and 5-14 days for enterococcal CRBSI $[13,14,16,19,20,32]$ (Table 3). As the treatment duration depends on several factors (e.g. host, causative pathogen, complications and the type of catheter), the decision on how long to treat a patient should be individualized. Further research to determine the optimum for each patient is urgently needed. In the interim, we discourage prolonged therapy for uncomplicated Gram-negative CRBSI (i.e. longer than 7 days) and we recommend considering very short treatment for uncomplicated CoNS-CRBSI after catheter removal. Longer antibiotic treatment durations may be needed for more compromised patients. Optimal duration of treatment for uncomplicated enterococcal CRBSI remains an unresolved issue and a treatment of 7-14 days appears to be reasonable [33]. 


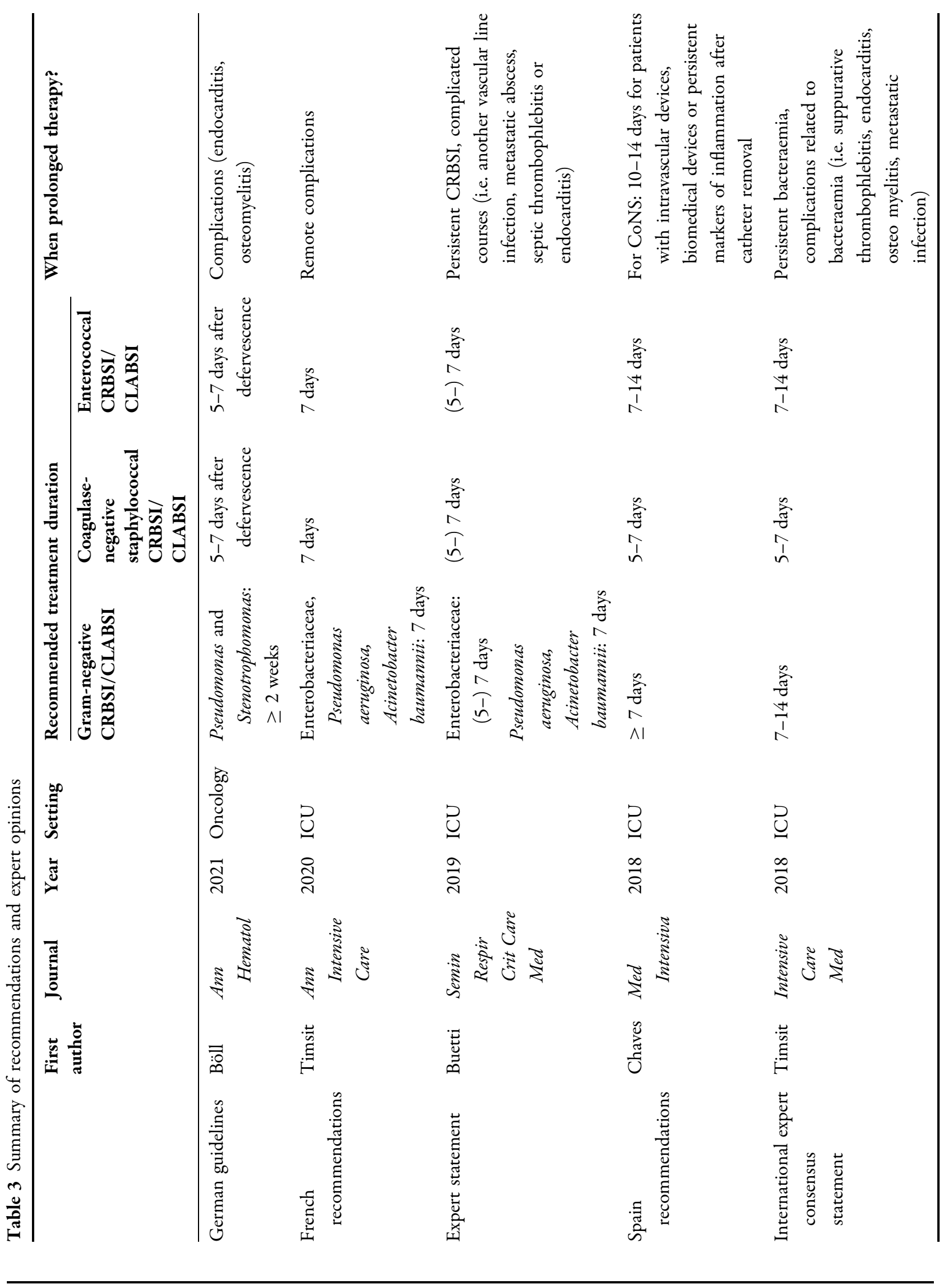




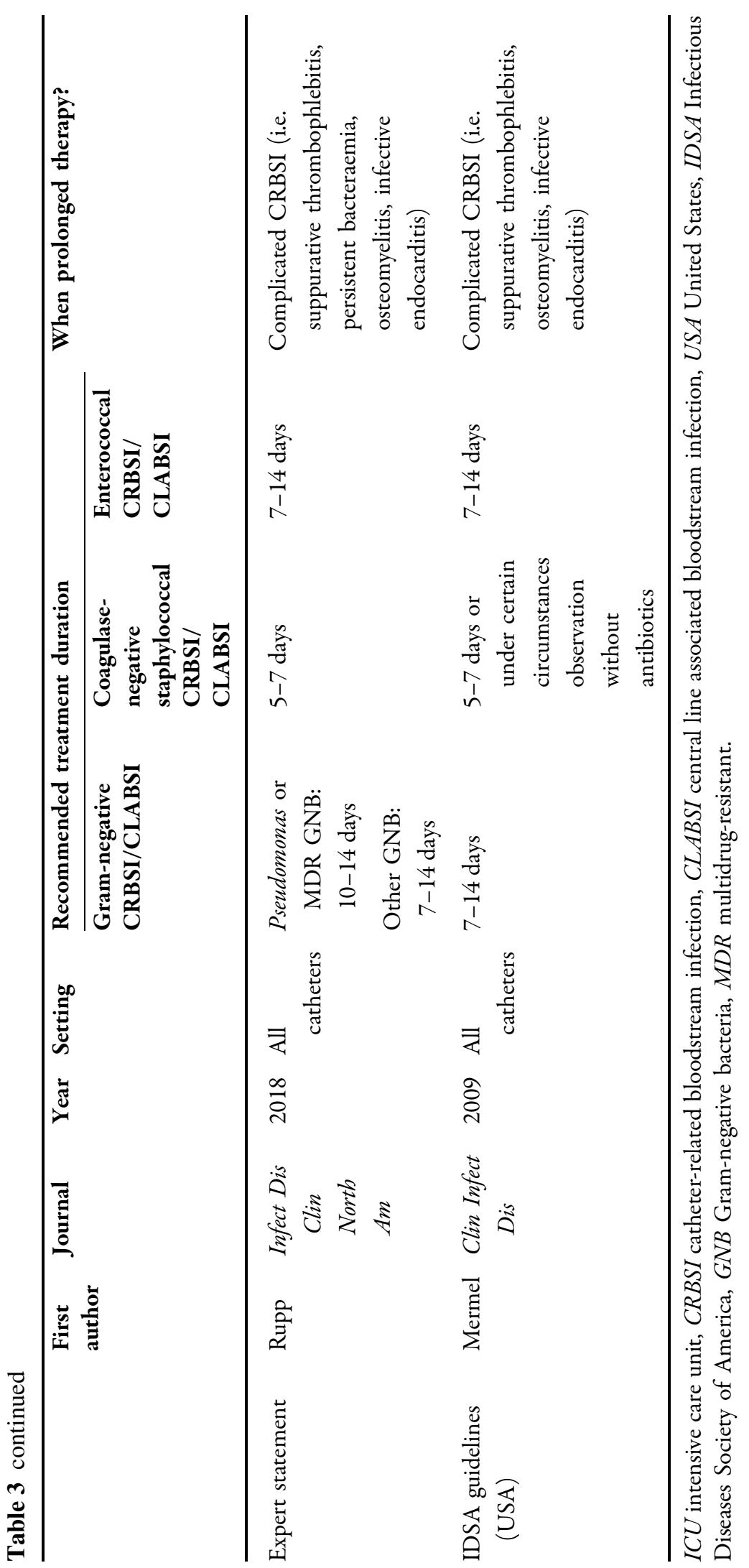


Our systematic review has several limitations. We did not perform a quantitative synthesis because of the heterogeneity and low quality of the studies. We modified our design compared to the published protocol by including studies which did not exclude paediatric patients but studies exclusively including paediatric patients were excluded. Paediatric represented a limited part of the included patients and recommendations for this specific population cannot be provided. Moreover, our outcome definition of clinical failure/cure was modified. Studies of enterococcal CRBSI did not perform a comparison between short- and longcourse treatment. As a consequence, an exact evaluation of treatment duration was not possible. We did not include studies using antimicrobial lock therapy which is recommended in guidelines and in use clinically at some institutions. We did not differentiate studies involving different central venous catheters (e.g. short-term catheters versus long-term catheters such as tunnelled or implanted catheter or those used exclusively for dialysis). We did not differentiate Gram-negative infections on the basis of the pathogen involved (e.g. outcomes of CRBSI due to Pseudomonas spp. versus Klebsiella spp). Finally, we included only uncomplicated CRBSI and therefore no conclusion can be drawn on complicated intravascular catheter infections.

\section{CONCLUSION}

Our review suggests that shorter antibiotic duration for uncomplicated intravascular catheter infections due to CoNS and Gramnegative bacilli can be implemented when the infected catheter was removed. However, welldesigned studies are urgently needed to confirm this recommendation.

\section{ACKNOWLEDGEMENTS}

Funding. Niccolò Buetti is currently receiving a Mobility grant from the Swiss National Science Foundation (Grant No.
P4P4PM_194449). The Journal's Rapid Service Fee was funded by Swiss National Science Foundation.

Authorship. All named authors meet the International Committee of Medical Journal Editors (ICMJE) criteria for authorship for this article, take responsibility for the integrity of the work as a whole, and have given their approval for this version to be published.

Authors' Contributions. Niccolò Buetti and Severin Muff contributed to the study conception and design. Niccolò Buetti and Severin Muff were responsible for the literature search and the data collection. All authors analysed and interpreted the data. Niccolò Buetti and Severin Muff were the major contributors in writing the manuscript. Alexis Tabah, Yok-Ai Que, Jean-François Timsit, Leonard Mermel and Stephan Harbarth critically revised the manuscript. All authors read and approved the final manuscript.

Disclosures. Severin Muff, Alexis Tabah, Yok-Ai Que, Stephan Harbarth, Niccolò Buetti have disclosed that they do not have conflict of interest. Jean-François Timsit received fees for lectures from 3M, MSD, Pfizer, and Biomerieux. Jean-François Timsit received research grants from Astellas, 3M, MSD, and Pfizer. Jean-François Timsit participated in advisory boards of 3M, MSD, Bayer Pharma, Nabriva, and Pfizer. Leonard Mermel has served as a consultant for Citius Pharmaceuticals, Marvao Medical, Leonard-Meron Biosciences, Destiny Pharma, and Nobio unrelated to the submitted work.

Compliance with Ethics Guidelines. This article is based on previously conducted studies and does not contain any new studies with human participants or animals performed by any of the authors.

Data Availability. The datasets generated during and/or analysed during the current study are available from the corresponding author on reasonable request. Preliminary data were submitted (abstract) to the ICPIC conference (Geneva, September 2021). 
Open Access. This article is licensed under a Creative Commons Attribution-NonCommercial 4.0 International License, which permits any non-commercial use, sharing, adaptation, distribution and reproduction in any medium or format, as long as you give appropriate credit to the original author(s) and the source, provide a link to the Creative Commons licence, and indicate if changes were made. The images or other third party material in this article are included in the article's Creative Commons licence, unless indicated otherwise in a credit line to the material. If material is not included in the article's Creative Commons licence and your intended use is not permitted by statutory regulation or exceeds the permitted use, you will need to obtain permission directly from the copyright holder. To view a copy of this licence, visit http://creativecommons.org/licenses/by$\mathrm{nc} / 4.0 /$.

\section{REFERENCES}

1. Centers for Disease Control and Prevention. Central line-associated bloodstream infections: A.R. \& Patient Safety Portal. 2019. https://arpsp.cdc.gov/ profile/infections/CLABSI. Accessed 11 Jan 2021.

2. European Centre for Disease Prevention and Control. Healthcare-associated infections in intensive care units - Annual Epidemiological Report for 2017. ECDC; 2019 Oct. https://www.ecdc.europa. eu/sites/default/files/documents/AER_for_2017HAI.pdf.

3. Dimick JB, Pelz RK, Consunji R, Swoboda SM, Hendrix CW, Lipsett PA. Increased resource use associated with catheter-related bloodstream infection in the surgical intensive care unit. Arch Surg. 2001;136:229.

4. Olaechea PM, Palomar M, Álvarez-Lerma F, et al. Morbidity and mortality associated with primary and catheter-related bloodstream infections in critically ill patients. Rev Esp Quimioter. 2013;26: 21-9.

5. Ziegler MJ, Pellegrini DC, Safdar N. Attributable mortality of central line associated bloodstream infection: systematic review and metaanalysis. Infection. 2015;43:29-36.

6. Zimlichman E, Henderson D, Tamir O, et al. Health care-associated infections: a meta-analysis of costs and financial impact on the US health care system. JAMA Intern Med. 2013;173:2039-46.

7. Novosad SA, Fike L, Dudeck MA, et al. Pathogens causing central-line-associated bloodstream infections in acute-care hospitals-United States, 2011-2017. Infect Control Hosp Epidemiol. 2020;41:313-9.

8. The Task Force for Combating Antibiotic-Resistant Bacteria. National Action Plan for Combating Antibiotic-Resistant Bacteria, 2020-2025. 2020. https://aspe.hhs.gov/pdf-report/carb-plan-20202025. Accessed 7 Jan 2021.

9. von Dach E, Albrich WC, Brunel A-S, et al. Effect of C-reactive protein-guided antibiotic treatment duration, 7-day treatment, or 14-day treatment on 30-day clinical failure rate in patients with uncomplicated Gram-negative bacteremia: a randomized clinical trial. JAMA. 2020;323:2160.

10. Yahav D, Franceschini E, Koppel F, et al. Seven versus 14 days of antibiotic therapy for uncomplicated Gram-negative bacteremia: a noninferiority randomized controlled trial. Clin Infect Dis. 2019;69:1091-8.

11. Tansarli GS, Andreatos N, Pliakos EE, Mylonakis E. A systematic review and meta-analysis of antibiotic treatment duration for bacteremia due to Enterobacteriaceae. Antimicrob Agents Chemother. 2019;63:e02495-e2518.

12. Li X, Liu C, Mao Z, Li Q, Qi S, Zhou F. Short-course versus long-course antibiotic treatment in patients with uncomplicated gram-negative bacteremia: a systematic review and meta-analysis. J Clin Pharm Ther. 2021;46:173-80.

13. Böll B, Schalk E, Buchheidt D, et al. Central venous catheter-related infections in hematology and oncology: 2020 updated guidelines on diagnosis, management, and prevention by the Infectious Diseases Working Party (AGIHO) of the German Society of Hematology and Medical Oncology (DGHO). Ann Hematol. 2021;100:239-59.

14. Timsit J-F, Baleine J, Bernard L, et al. Expert consensus-based clinical practice guidelines management of intravascular catheters in the intensive care unit. Ann Intensive Care. 2020;10:118.

15. Mermel LA, Allon M, Bouza E, et al. Clinical practice guidelines for the diagnosis and management of intravascular catheter-related infection: 2009 update by the Infectious Diseases Society of America. Clin Infect Dis. 2009;49:1-45.

16. Chaves F, Garnacho-Montero J, Del Pozo JL, et al. Diagnosis and treatment of catheter-related bloodstream infection: clinical guidelines of the Spanish 
Society of Infectious Diseases and Clinical Microbiology and (SEIMC) and the Spanish Society of Spanish Society of Intensive and Critical Care Medicine and Coronary Units (SEMICYUC). Med Intens. 2018;42:5-36.

17. Moher D, Liberati A, Tetzlaff J, Altman DG, PRISMA Group. Preferred reporting items for systematic reviews and meta-analyses: the PRISMA statement. PLoS Med. 2009;6:e1000097.

18. Ouzzani M, Hammady H, Fedorowicz Z, Elmagarmid A. Rayyan-a web and mobile app for systematic reviews. Syst Rev. 2016;5:210.

19. Rupp ME, Karnatak R. Intravascular catheter-related bloodstream infections. Infect Dis Clin $\mathrm{N}$ Am. 2018;32:765-87.

20. Buetti N, Timsit J-F. Management and prevention of central venous catheter-related infections in the ICU. Semin Respir Crit Care Med. 2019;40:508-23.

21. Wells G, Shea B, O'Connell D, et al. The Newcastle-Ottawa Scale (NOS) for assessing the quality of non-randomized studies in meta-analysis. 2000. http://www.ohri.ca/programs/clinical_ epidemiology/oxford.asp. Accessed 19 Jan 2021.

22. Ruiz-Ruigómez M, Fernández-Ruiz M, San-Juan R, et al. Impact of duration of antibiotic therapy in central venous catheter-related bloodstream infection due to Gram-negative bacilli. J Antimicrob Chemother. 2020;75:3049-55.

23. San-Juan R, Martínez-Redondo I, Fernández-Ruiz M, et al. A short course of antibiotic treatment is safe after catheter withdrawal in catheter-related bloodstream infections due to coagulase-negative staphylococci. Eur J Clin Microbiol Infect Dis. 2019;38:977-83.

24. Lee Y-M, Moon C, Park K-H. Is short-term antibiotic therapy safe in patients with central venous catheter-related Gram-negative bacteraemia? J Antimicrob Chemother. 2021;76:539-41.

25. Sandoe JAT, Witherden IR, Au-Yeung H-KC, Kite P, Kerr KG, Wilcox MH. Enterococcal intravascular catheter-related bloodstream infection: management and outcome of 61 consecutive cases. J Antimicrob Chemother. 2002;50:577-82.

26. Marschall J, Piccirillo ML, Fraser VJ, Doherty JA, Warren DK. Catheter removal versus retention in the management of catheter-associated enterococcal bloodstream infections. Can J Infect Dis Med Microbiol. 2013;24:e83-7.

27. Hebeisen UP, Atkinson A, Marschall J, Buetti N. Catheter-related bloodstream infections with coagulase-negative staphylococci: are antibiotics necessary if the catheter is removed? Antimicrob Resist Infect Control. 2019;8:21.

28. Raad I, Kassar R, Ghannam D, Chaftari AM, Hachem R, Jiang Y. Management of the catheter in documented catheter-related coagulase-negative staphylococcal bacteremia: remove or retain? Clin Infect Dis. 2009;49:1187-94.

29. Surapat B, Montakantikul P, Malathum K, Kiertiburanakul S, Santanirand P, Chindavijak B. Microbial epidemiology and risk factors for relapse in Gram-negative bacteria catheter-related bloodstream infection with a pilot prospective study in patients with catheter removal receiving short-duration of antibiotic therapy. BMC Infect Dis. 2020;20:604.

30. Centers for Disease Control and Prevention. National and State Associated Infections Progress Report. Centers for Disease Control and Prevention. 2016. https://www.cdc.gov/HAI/pdfs/progressreport/hai-progress-report.pdf.

31. Heilmann C, Ziebuhr W, Becker K. Are coagulasenegative staphylococci virulent? Clin Microbiol Infect. 2019;25:1071-80.

32. Timsit J-F, Rupp M, Bouza E, et al. A state of the art review on optimal practices to prevent, recognize, and manage complications associated with intravascular devices in the critically ill. Intensive Care Med. 2018;44:742-59.

33. Muff S, Hebeisen U, Timsit J-F, Mermel L, Harbarth $\mathrm{S}$, Buetti N. Treatment duration of enterococcal intravascular catheter-related infections. Clin Microbiol Infect. 2020;S1198-743X:30717-5. 